\title{
Unruptured ectopic pregnancy in rudimentary horn of unicornuate uterus at fourteen weeks with previous vaginal delivery
}

\author{
Jaydip Hindocha ${ }^{1}$, Sneh Sonaiya ${ }^{2 *}$
}

${ }^{1}$ Department of Obstetrics and Gynecology, ${ }^{2}$ SSG Hospital and Medical College, Baroda, Gujarat, India

Received: 19 September 2020

Accepted: 31 October 2020

\section{*Correspondence:}

Dr. Sneh Sonaiya,

E-mail: snehsonaiya123@gmail.com

Copyright: (C) the author(s), publisher and licensee Medip Academy. This is an open-access article distributed under the terms of the Creative Commons Attribution Non-Commercial License, which permits unrestricted non-commercial use, distribution, and reproduction in any medium, provided the original work is properly cited.

\begin{abstract}
Unicornuate uterus with a rudimentary horn is an anomaly of the mullerian duct and is an extremely rare condition. This condition results when one of the paired mullerian ducts fails to fuse completely. Its incidence is estimated to be one in 76,000 pregnancies. Pregnancy in the rudimentary horn of the unicornuate uterus is difficult to diagnose on ultrasound and can be easily missed out. Hence the pregnancy usually gets detected after rupture when the mother presents with the complaint of severe abdominal pain. This is a case report of a 24 year old $\mathrm{G}_{2} \mathrm{P}_{1} \mathrm{~A}_{0} \mathrm{~L}_{1}$ female who presented to us with complaints of tenderness in the left iliac fossa and mild abdominal distention. Clinical examinations, radiological investigations, and exploratory laparotomy revealed a unicornuate uterus with an unruptured left rudimentary horn pregnancy at 14 weeks with mild hemoperitoneum. Following the exploratory laparotomy, excision of the left rudimentary horn and thorough peritoneal lavage was performed.
\end{abstract}

Keywords: Rudimentary horn pregnancy, Unicornuate uterus, Mullerian anomaly

\section{INTRODUCTION}

As the embryo develops, two mullerian ducts form the female reproductive system, which includes the fallopian tubes, uterus, cervix, and the upper two-thirds of the vagina. Mullerian malformations result when there is a defective development or fusion of these ducts. A wide range of anomalies can result due to the disruption of the mullerian duct system, ranging from agenesis of the vagina to the duplication of the cervix and vagina. Based on the degree of an anomaly, mullerian duct anomalies (MDAs) are classified into a seven class system. A unicornuate uterus is a type $2 \mathrm{MDA}$ and can be further classified into communicating, non-communicating, no cavity, and no horn. ${ }^{1}$ Rudimentary horn pregnancy can lead to several obstetric complications, most commonly causing massive hemoperitoneum and shock. According to the available literature, the newborn survival rate is between $0 \%$ and $13 \%$ in the rudimentary horn pregnancies with only one-third of such gestations reaching term or beyond. More than $50 \%$ of the pregnancies ended with a rupture of the pregnant uterine horn. $^{2}$

\section{CASE REPORT}

A 24 year old female presented to us with the complaints of amenorrhoea for three months and tenderness in the left iliac fossa and mild abdominal distention for two days. The patient was referred to us from a rural private set-up that suspected the female to have extra-uterine pregnancy in the left adnexa. The patient had a previous uneventful vaginal delivery of a $2.5 \mathrm{~kg}$ baby 2 years back and this was her second pregnancy. Repeated ultrasonography of the abdomen and pelvis was suggestive of mild hemoperitoneum with clots in the endometrial cavity. On clinical examination, the patient had feeble pulse. Her abdomen was tense and distended 
and the uterine size was not made out. Pelvic examination revealed fullness in the fornices with severe left iliac fossa tenderness. The patient was taken for emergency laparotomy.

Emergency laparotomy was performed for uterine exploration suspecting a ruptured ectopic pregnancy. Exploratory laparotomy revealed an unruptured left rudimentary non-communicating horn of a unicornuate uterus (Figure 1) with the fetus and intact sac lying free in the peritoneal cavity with a mild hemoperitoneum.

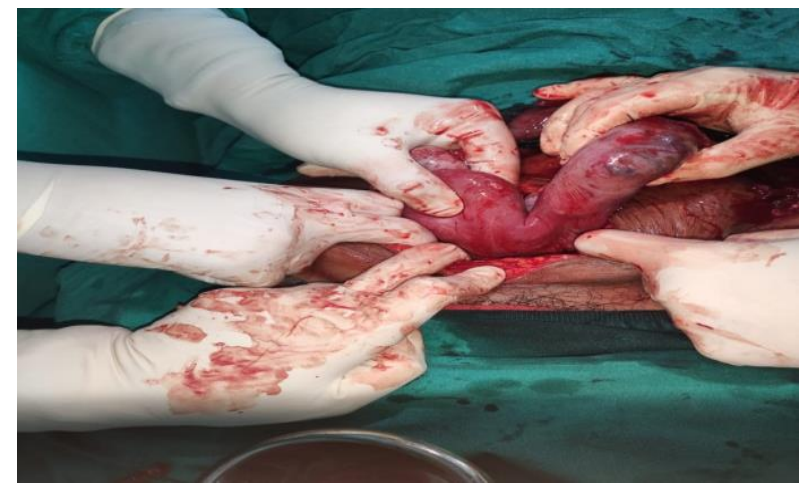

Figure 1: Unruptured left rudimentary noncommunicating horn of a unicornuate uterus.

The fetus was of approximately 14 weeks and 3 days, with the unicornuate uterus (Figure 2). After the exploratory laparotomy, the rudimentary horn was excised and thorough peritoneal lavage was performed. The patient required one pint of PCV transfusion postoperatively. The microbiological examinations of the patient revealed 10-12 pus cells per high power field, suggesting a urinary tract infection. Henceforth, the patient was kept on higher antibiotics and was discharged from the hospital on the eighth postoperative day.
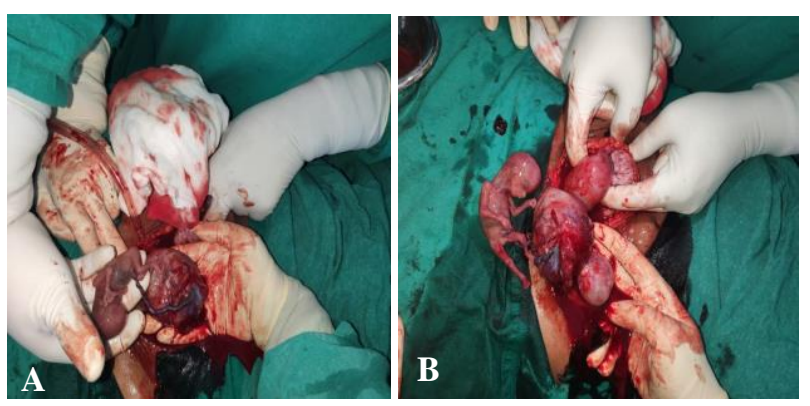

Figure 2: A) Fourteen weeks old fetus B) From the unruptured horn of the uterus.

\section{DISCUSSION}

The parts of the female reproductive system that develop from the mullerian ducts include fallopian tubes, uterus, cervix, and the upper two-thirds of the vagina. Mullerian anomalies result due to incomplete or improper fusion of the mullerian ducts. A unicornuate uterus with a rudimentary horn develops due to the failure of the development of one mullerian duct and failure of fusion with the opposite side. Rudimentary horn pregnancy in a unicornuate uterus is a rare clinical condition with a reported incidence of 1 in 100,000 to 140,000 pregnancies. ${ }^{3}$ A rudimentary horn of the unicornuate uterus may be of the communicating or the noncommunicating type. Pregnancy in the rudimentary horn of the unicornuate uterus occurs via transperitoneal migration of the spermatozoon or the transperitoneal migration of the fertilized ovum. ${ }^{4}$ Such pregnancies usually do not result in a viable child. Horn pregnancy usually ruptures between the second and third trimester, most commonly between the 10th and 20th weeks of gestation. The rudimentary horn usually ruptures due to underdeveloped myometrium and a dysfunctional endometrium. ${ }^{5}$ Because of lack of awareness about mullerian anomalies, early diagnosis can be easily missed. Hence a deviated uterus with a palpable adnexal mass on pelvic examination in the first trimester should arouse a suspicion of mullerian anomaly. Ultrasound, hysterosalpingogram, hysteroscopy, laparoscopy, and MRI can be useful diagnostic tools. The rudimentary horn with a unicornuate uterus can also be associated with unilateral renal anomalies. ${ }^{6}$

In the case of this patient, the diagnosis of a rudimentary horn pregnancy was missed in the first ultrasound scan. It was only when the patient presented with left iliac fossa tenderness and a distended abdomen that ectopic pregnancy was suspected and emergency laparotomy revealed the unruptured horn pregnancy. This case highlights the importance of emergency surgery in suspected ectopic pregnancies. In the case of confirmed rudimentary horn pregnancies with early gestational age, laparoscopic surgery, systemic methotrexate administration, or feticide with intracardiac potassium chloride have been recommended as alternatives to laparotomy.

\section{CONCLUSION}

Due to the lack of advanced ultrasound techniques in developing countries, the pregnancy in the rudimentary horn is difficult to diagnose. The patient usually presents with a ruptured rudimentary horn and massive hemoperitoneum. Additionally, the diagnosis can easily be missed by inexperienced doctors, and the morbidity of the patient increases due to misdiagnosis and loss of time in transferring the patient. Henceforth, increased awareness about mullerian anomalies and its radiological features amongst the physicians working in peripheral set-ups can be of prime importance. Timely diagnosis, resuscitation, exploratory laparotomy, and blood transfusion are needed to reduce morbidity and mortality in the cases of rudimentary horn ectopic pregnancies.

Funding: No funding sources Conflict of interest: None declared Ethical approval: Not required 


\section{REFERENCES}

1. Hassan CH, Karim AK, Ismail NA, Omar MH. Case report of ruptured non-communicating right rudimentary horn pregnancy: an acute emergency. Acta Medica (Hradec Kralove). 2011;54(3):125-6.

2. Ambusaidi Q, Jha C. Pregnancy in the Rudimentary Uterine Horn: Case report of an unusual presentation. Sultan Qaboos Univ Med J. 2014;14(1):e134-8.

3. Chang JC, Lin YC. Rupture of rudimentary horn pregnancy. Acta Obstet Gynecol Scand. 1992;71(3): 235-8.

4. Scholtz M. A full-time pregnancy in a rudimentary horn of the uterus. Brit J Obstet Gynaecol. 1951;58: 293-6.

5. Jain R, Gami N, Puri M, Trivedi S. A rare case of intact rudimentary horn pregnancy presenting as hemoperitoneum. J Hum Reprod Sci. 2010;3(2):1135.
6. Henriet E, Roman H, Zanati J, Lebreton B, Sabourin JC, Loic M. Pregnant noncommunicating rudimentary uterine horn with placenta percreta. J Soc Laparosc Robot Surg. 2008;12(1):101-3.

Cite this article as: Hindocha $\mathrm{J}$, Sonaiya S. Unruptured ectopic pregnancy in rudimentary horn of unicornuate uterus at fourteen weeks with previous vaginal delivery. Int J Reprod Contracept Obstet Gynecol 2020;9:5166-8. 